\title{
Safe Delivery Solutions for COVID-19 Infected Pregnancies
}

\author{
Hongbo Qi ${ }^{1}$, Xin Luo ${ }^{2}$, Yangxi Zheng ${ }^{3}$, Hua Zhang ${ }^{4}$, Jiafu $\mathrm{Li}^{5}$, Li Zou ${ }^{6}$, Ling Feng ${ }^{7}$, Dunjin \\ Chen $^{8}$, Yuan $\mathrm{Shi}^{1}$, Chao Tong ${ }^{2}$, and Philip Baker ${ }^{9}$ \\ ${ }^{1}$ Chongqing Medical University Affiliated Children's Hospital \\ ${ }^{2}$ Affiliation not available \\ ${ }^{3}$ University of Texas-Houston Medical School \\ ${ }^{4}$ The First Affiliated Hospital of Chongqing Medical University, Canada-China-New \\ Zealand Joint Laboratory of Maternal and Fetal Medicine, Chongqing Medical University \\ ${ }^{5}$ Wuhan University Zhongnan Hospital \\ ${ }^{6}$ Huazhong University of Science and Technology Tongji Medical College \\ ${ }^{7}$ Tongji Hospital of Tongji Medical College of Huazhong University of Science and \\ Technology \\ ${ }^{8}$ Third Affiliated Hospital of Guangzhou Medical University \\ ${ }^{9}$ University of Leicester
}

May 5, 2020

Since December 2019, a new coronavirus (COVID-19) infection has rapidly become prevalent in central China [1]. On the basis of knowledge obtained from a previous coronavirus outbreak [2], pregnant women are believed to be susceptible to this virus. Once a maternal infection of $2019-\mathrm{nCoV}$ is suspected or confirmed, childbirth becomes complicated and challenging. Efficient obstetric treatment is required, and is key to optimizing the prognosis for both mother and child. Care should be taken in determination of the timing of delivery, assessment of the indications for caesarean section, preparation of the delivery room to prevent infection, choice of the type of anesthesia, and newborn management.

Timing of delivery

According to the Expert Advice on Novel Coronavirus Infection in Pregnancy and Puerperium [1, 3], issued by Chinese Medical Association (CMA), the current consensus is that 2019-nCoV infection is not an absolute indication for ending pregnancy, but that expedition of delivery should be evaluated on a case-by-case basis. Maternal disease progression, gestational age and fetal intrauterine status are primary concerns. If maternal safety is assured, the timing of delivery should primarily be determined by the gestational age.

After consulting with several obstetric experts in Wuhan city, who have each dealt with COVID-19 infected pregnancies, we suggest that the timing of delivery for COVID-19 infection complicated pregnancies should be based on the following four principles:

1. If the infected pregnant women demonstrate obstetric indications for early delivery, such as placenta previa, preeclampsia, malpresentation etc., the timing of delivery should be based on the specific obstetric circumstances.

2. If the infection of COVID-19 is not improved by treatment, early delivery should be considered, even in the absence of obstetric indications.

3. If the maternal COVID-19 infection is assessed as severe or critical, according to the diagnostic criteria in the National Health and Medical Commission's New Coronavirus Infection Pneumonia Diagnosis and 
Treatment Program (the fifth edition), early delivery needs to be considered to ensure maternal safety, regardless of gestational age [4].

(a) Severe: Respiratory distress (RR[?]30 beats / min), or mean oxygen saturation [?]93\% at rest, or arterial blood oxygen partial pressure (PaO2) / oxygen concentration (FiO2) [?]300mmHg;

(b) Critical: Respiratory failure and mechanical ventilation required, or shock, or combined with other organ failure and requires ICU monitoring and treatment.

4. Whether mild or common COVID-19 infection is an indication for delivery in the third trimester remains to be determined. During severe epidemics, delivery after 32-34 gestational weeks may be beneficial to the subsequent treatment and safety of these patients [5].

In summary, the current opinion of obstetric experts is that timing of delivery should be determined by the maternal disease status. Maternal safety is the priority; multidisciplinary consultation and the opinions of critical care medical experts should be considered carefully.

Indications for caesarean section

The determination of mode of delivery should be based on obstetric indications. However, the safety of vaginal birth, cesarean section or other methods in the context of COVID-19 infection has yet been confirmed [6]. Nevertheless, senior obstetricians in Wuhan suggest two recommendations:

1. During the current period of emergency [7], the indications for caesarean section for women with COVID19 infection should be applied flexibly and the threshold for Caesarean section lowered;

2. In particular, the threshold for caesarean section on the basis of delay in the first stage of labour should be lowered;

These suggestions are aimed at reducing maternal in-patient stays, minimizing the chance of cross-infection, reducing maternal physical exertion during delivery and ensuring the safety of other postnatal women, newborns and health care workers. There are currently very few COVID-19 infected pregnant women, thus a lowered threshold for caesarean section will not significantly increase the overall caesarean section rate.

Delivery room and infection protection

1. Delivery should take place on a negative pressure isolation ward [3];

2. If a negative pressure isolation ward is unavailable, then an infection isolation ward or infection isolation surgery room should be used for delivery. A regular delivery room should not be used;

3. Unnecessary items should be removed from the delivery room, and the number of medical and other attending staff minimized (2-3 skilled obstetricians and midwives/nurses are sufficient);

4. Strict infection protection for all attending staff must be adhered to throughout the entire delivery process, including vaginal examinations, artificial rupture of fetal membranes, etc. This entails the use of disposable hats and protective clothing, shoe covers, N95 masks, goggles/face shields and two-layer gloves which can cover the sleeves of protective clothing. Hands should be washed in strict accordance with the Seven-steps Hand Washing Method and then disinfected by the use of alcohol or hydrogen peroxide disinfection liquid $[8]$

6. Patients with non-general anesthesia should wear surgical masks during delivery.

Anesthesia

Epidural anesthesia or general anesthesia can be used for delivery in pregnant women infected with pneumonia of COVID-19 [3, 9]; for those COVID-19 infected pregnant women who already have had intubation, general anesthesia through endotracheal intubation can be used during the cesarean section. 
Most practitioners advocate the use of epidural anesthesia for pregnant women infected with pneumonia of COVID-19 to reduce exacerbation of infection during the intubation/extraction process, and to avoid the side effects of general anesthesia on newborns (muscle tone and breathing). Importantly, anesthesiologists must apply airborne precautions to prevent infection during performing trachea intubation.

Neonatal management

1. Obstetricians and pediatricians should cooperate closely; the neonatologist should be notified at least 30 minutes in advance of any planned delivery, in order to attend and make any necessary preparations;

2. Newborns of the suspected COVID-19 infected mother should be transferred to the neonatal isolation ward immediately after being evaluated as in good general condition by the attending neonatologist. If the maternal nucleic acid test is negative for 2 consecutive tests, the newborn may be transferred out of the isolation ward for mother-to-child or home care [3];

3. If the maternal nucleic acid test is positive or if a maternal infection is confirmed, newborns should be quarantined for at least 14 days [5].

Points to note

1. Caesarean section should be performed by a senior obstetrician, in order to minimize the likelihood of complications;

2. During the caesarean section, careful attention should be paid achieving hemostasis, to prevent postpartum hemorrhage and risk of challenging blood transfusion;

3. Biospecimen including vaginal secretions, umbilical blood, amniotic fluid, placenta, neonatal throat swab should be collected during delivery to determine the potential intrauterine vertical transmission of COVID19 ;

3. Following delivery, all surgical instruments should be labeled "COVID-19", and then stored, transported and sterilized in processes separate from other instruments;

4. Medical and midwifery staff who participate in vaginal birth or cesarean section of COVID-19 infected mothers should be followed up and given body temperature monitoring. If any of the signs of infection occur, including but not limited to fever, cough, fatigue, nasal congestion, runny nose, diarrhea and other manifestations, potentially affected staff should attend the fever clinic of a designated hospital as soon as possible, in order that a definitive diagnosis be obtained.

\section{Funding}

None.

\section{Ethics Approval}

Not required.

\section{Contribution to Authorship}

H.Q, X.L, Y.Z, H.Z, J.L, L.Z, L.F, D.C, Y.S, C.T, and P. B conceived this study, H.Q, X.L, Y.Z, C.T, and P. B wrote the draft, H.Z, J.L, L.Z, L.F, D.C, and Y.S revised the manuscript.

\section{Disclosure of Interests}

None declared.

\section{References}

[1] World Health Organization. Clinical management of severe acute respiratory infection when novel coronavirus (nCoV) infection is suspected:Interim guidance;[EB/OL].(2020-01-28)[2020-01 29].12 Jan- 
uary 2020; WHO/nCoV/Clinical/2020.1 https://www.who.int/publications-detail/clinical-management-ofsevere-acute-respiratory-infection-when-novel-coronavirus-(ncov)-infection-is-suspected

[2] Jamieson DJ, Honein MA, Rasmussen S.A, et al. H1N1 2009 influenza virus infection during pregnancy in the USA. Lancet, 2009,374(9688) :429-430.

[3] Maxwell C, McGeer A, KFY T, et al. No. 225-Management guidelines for obstetric patients and neonates born to mothers with suspected or probable severe acute respiratory syndrome (SARS) [J]. J Obstet Gynaecol Can, 2017,39(8):e130-e137.

[4] Huang C, Wang Y, Li X, et al. Clinical features of patients infected with 2019 novel coronavirus in Wuhan, China. Lancet. 2020, S0140-6736(20)30183-5.DOI: 10.1016/S0140-6736(20)30154-9

[5] Wang J, Qi H, Bao L, et al. A contingency plan for the management of the 2019 novel coronavirus outbreak in neonatal intensive care units. Lancet Child Adolesc Health 2020; published online Feb 7 https://doi.org/10.1016/S2352-4642(20)30040-7

[6] Julien R, Christian AL. Pattern of early human-to-human transmission of Wuhan 2019 novel coronavirus (2019-nCoV), December 2019 to January 2020. Euro Surveill. 2020;25(4):pii=2000058. https://doi.org/10.2807/1560-7917.ES.2020.25.4.2000058

[7] Lancet T. Emerging understandings of 2019-nCoV. Lancet, 2020, Jan 24.pii.S0140-6736 (20)30186-0. DOI: $10.1016 /$ S0140-6736(20)30186-0.

[8] Office of the National Health Commission, Office of the National Administration of Traditional Chinese Medicine. Guidelines for the Diagnosis and Treatment of Novel Coronavirus (2019-nCoV) Infection (Interim Version 5, the Revision). National Health Commission Bulletin NO. 2020(117). [EB/OL].[2020-0208].http://www.gov.cn/zhengce/zhengceku/2020-02/09/content_5476407.htm

[9] Rochwerg B, Brochard L, Elliott MW, et al. Official ERS/ATS clinical practice guidelines: noninvasive ventilation for acute respiratory failure. Eur Respir J, 2017, 50(2). pii:1602426. DOI:10.1183/13993003.02426-2016. 